\title{
The Efficacy of Hypnotherapy in the Treatment of Irritable Bowel Syndrome: A Systematic Review and Meta-analysis
}

\author{
Han Hee Lee, ${ }^{1}$ Yoon Young Choi ${ }^{2}$ and Myung-Gyu Choi ${ }^{1 *}$ \\ ${ }^{1}$ Division of Gastroenterology, Department of Internal Medicine, College of Medicine, The Catholic University of Korea, Seoul, Korea; and \\ ${ }^{2}$ Department of Surgery, Yonsei University College of Medicine, Seoul, Korea
}

\begin{abstract}
Background/Aims
Hypnotherapy is considered as a promising intervention for irritable bowel syndrome (IBS), but the evidence is still limited. The aims of this study were to conduct a systematic review and meta-analysis to estimate the efficacy of hypnotherapy for the treatment of IBS.
\end{abstract}

\section{Methods}

A literature search was performed using MEDLINE (PubMed), Embase, PsycINFO and the Cochrane Central Register of Controlled Trials (CENTRAL database). Only randomized controlled trials that compared hypnotherapy with any other conventional treatment or no treatment in patients with IBS were included. Studies had to report outcomes as IBS symptom score or quality of life. The mean change in outcome score was used to pool these outcomes for the meta-analysis. Data were synthesized using the standardized mean difference for continuous data.

\section{Results}

Seven randomized controlled trials (6 papers) involving 374 patients with IBS were identified. Performance bias was high in all trials because it was impossible to blind participants and therapists in this type of intervention. The outcomes in this meta-analysis were evaluated at 3 months for short-term effects and at 1 year for long-term effects. The change in abdominal pain score at 3 months was significant in the hypnotherapy group (standardized mean difference, $-0.83 ; 95 \% \mathrm{Cl},-1.65$ to -0.01 ). Three of the 4 trials showed greater improvement in overall gastrointestinal symptoms in the hypnotherapy group.

\section{Conclusions}

This study provides clearer evidence that hypnotherapy has beneficial short-term effects in improving gastrointestinal symptoms of patients with IBS.

\section{(J Neurogastroenterol Motil 2014;20:152-162)}

Key Words

Gastrointestinal diseases; Hypnosis; Irritable bowel syndrome; Meta-analysis; Review

Received: September 5, 2013 Revised: November 27, 2013 Accepted: December 9, 2013

(c) This is an Open Access article distributed under the terms of the Creative Commons Attribution Non-Commercial License (http://creativecommons. org/licenses/by-nc/3.0) which permits unrestricted non-commercial use, distribution, and reproduction in any medium, provided the original work is properly cited.

*Correspondence: Myung-Gyu Choi, MD, PhD

Division of Gastroenterology, Department of Internal Medicine, Seoul St. Mary's Hospital, The Catholic University of Korea, 222 Banpo-daero, Seocho-Gu, Seoul 137-701, Korea

Tel: +82-2-2258-6017, Fax: +82-2-2258-2089, E-mail: choim@catholic.ac.kr

Financial support: None.

Conflicts of interest: None.

Han Hee Lee and Yoon Young Choi contributed equally to this work and joint with first author.

ORCID: Han Hee Lee, http://orcid.org/0000-0002-8244-374X; Myung-Gyu Choi, http://orcid.org/0000-0003-4083-5187. 


\section{Introduction}

Irritable bowel syndrome (IBS) is a chronic functional gastrointestinal disorder characterized by recurrent abdominal pain or discomfort associated with altered bowel movements. ${ }^{1}$ IBS can be diagnosed only when there is no objective evidence of an underlying organic disorder. ${ }^{2}$ It is one of the most common bowel disorders diagnosed by gastroenterologists. ${ }^{3}$ The impact of IBS on quality of life (QOL) is as strong as that observed in other serious disorders such as congestive heart failure or chronic renal failure. $^{4,5}$

Treatment options include reassurance, dietary modification, and pharmacological treatment. Current pharmacological treatments such as bulking agents, antispasmodics, and antidepressants focus mainly on controlling the symptoms of IBS. However, both pharmacological and conventional options are unsatisfactory in general. $^{6-8}$

Many IBS patients have psychological symptoms such as anxiety and depression, ${ }^{9}$ which provide a fundamental rationale for psychological treatment. A number of studies have been conducted to evaluate the effects of psychological treatment for IBS. ${ }^{10}$ Among them, hypnotherapy has gained popularity after the first randomized controlled trial (RCT) in 1984 demonstrating notable benefits of hypnotherapy. ${ }^{11}$ Several additional studies have also reported benefits of hypnotherapy in treating gastrointestinal symptoms and QOL of IBS patients. ${ }^{12-16}$

Previous systematic reviews to prove that there are statistically significant benefits have been based on pooled results of research on the effectiveness of hypnotherapy for IBS patients. ${ }^{17,18}$ These reviews concluded that, although a number of studies have shown a beneficial effect of hypnotherapy, insufficient evidence existed to recommend widespread use because of limitations in the sample sizes and methodological flaws. From these conclusions, the National Institute for Health and Clinical Excellence guidelines noted that hypnotherapy is a promising intervention for IBS but the evidence remains to be limited. ${ }^{19}$ These uncertainties justify a systematic review and meta-analysis to determine whether hypnotherapy has significant beneficial effects in the treatment of IBS. Well-designed RCTs have been conducted, but there is no ongoing RCT; this prompted us to update the previous review. We conducted a comprehensive review of RCTs to estimate the efficacy of hypnotherapy for the treatment of IBS.

\section{Materials and Methods}

\section{Eligibility Criteria}

The inclusion criteria in this study were specified in advance and followed the population, intervention, comparison and outcome(s) (PICO) study design: patients who had been diagnosed with IBS as the population of interest, given hypnotherapy as an intervention, or given any other conventional treatment or no treatment for comparison. Outcomes were overall gastrointestinal symptoms, individual gastrointestinal symptoms (abdominal pain, constipation and diarrhea) and QOL, which were measured as scores. Any studies that reported at least one of these outcomes were included. Only RCTs were included in this meta-analysis to provide more unbiased information than that obtained from other study designs. There was no limitation on publication language, study size or study setting. Patients were not otherwise restricted by age or ethics. Studies that included a comparison between different types of hypnotherapy were excluded.

\section{Search Strategy}

A literature search was conducted using MEDLINE (PubMed), Embase, PsycINFO, and the Cochrane Central Register of Controlled Trials (CENTRAL database) from January 15, 2013. The search was performed using the Medical Subject Headings (MeSH): "colonic diseases," "colonic diseases, functional," "irritable bowel syndrome," and "hypnosis." Other free-text search terms used were "irritable bowel syndrome," "hypnotherapy," "hypnosis," "mesmerism," "imagery" and "autohypnosis." Search terms were adapted according to each particular database. The strategy was refined further by a local health care librarian to ensure a good balance of sensitivity and specificity. Electronic searches were supplemented by manually searching the bibliographies of eligible clinical trials and previous systematic reviews.

\section{Study Selection and Data Extraction}

Two authors (H.H.L. and Y.Y.C.) independently screened the titles and abstracts of the papers identified by the initial search for relevance to this review. We retrieved the full text for any citation deemed potentially eligible at this stage. Two authors then separately assessed full articles using predetermined inclusion criteria to exclude irrelevant articles. Any disagreements regarding study inclusion were resolved by discussion. Data from the in- 
cluded studies were extracted by 2 authors to a Microsoft Excel spreadsheet (XP Professional; Microsoft Corp, Redmond, WA, USA). Conflicts in data extraction were resolved by consensus after referring back to the original article.

Searches of 4 of the electronic bibliographic databases initially identified 139 potentially relevant citations. Two additional studies were found by cross-referencing. Our search strategy identified 98 citations after removing duplicates, 30 of which were retrieved for full-text assessment, and a total of 7 RCTs ultimately fulfilled our inclusion criteria (Fig. 1). ${ }^{11,20-24}$ One study reported $2 \mathrm{RCT}$ s being conducted on different settings in one publication. ${ }^{20}$ One of the studies that we initially excluded was published only in abstract form. As we were conducting our meta-analysis, advance online publication of this study was reported in February 2013, so we have included it in our review. ${ }^{24}$ Agreement between authors for trial eligibility was substantial $(k$ statistic $=0.79)$.

\section{Quality Assessment}

Two authors independently assessed the methodological quality of the included trials using the risk of bias tool recommended by the Cochrane Collaboration. ${ }^{25}$ This included random sequence generation, allocation concealment, blinding of participants and personnel, blinding of outcome assessment, assessment of incomplete data outcome, selective reporting and other sources of bias. For each trial, the risk of bias was reported as "low risk," "unclear risk" or "high risk." Disagreement was resolved by discussion.

\section{Data Analysis}

All the assessed outcomes were continuous variables. Although the studies included in our review reported similar outcomes, various measurement instruments were applied to calculate the scores. The mean change in the outcome score was calculated and used to compare these outcomes by subtracting the baseline score from the score after treatment. The standard devia-

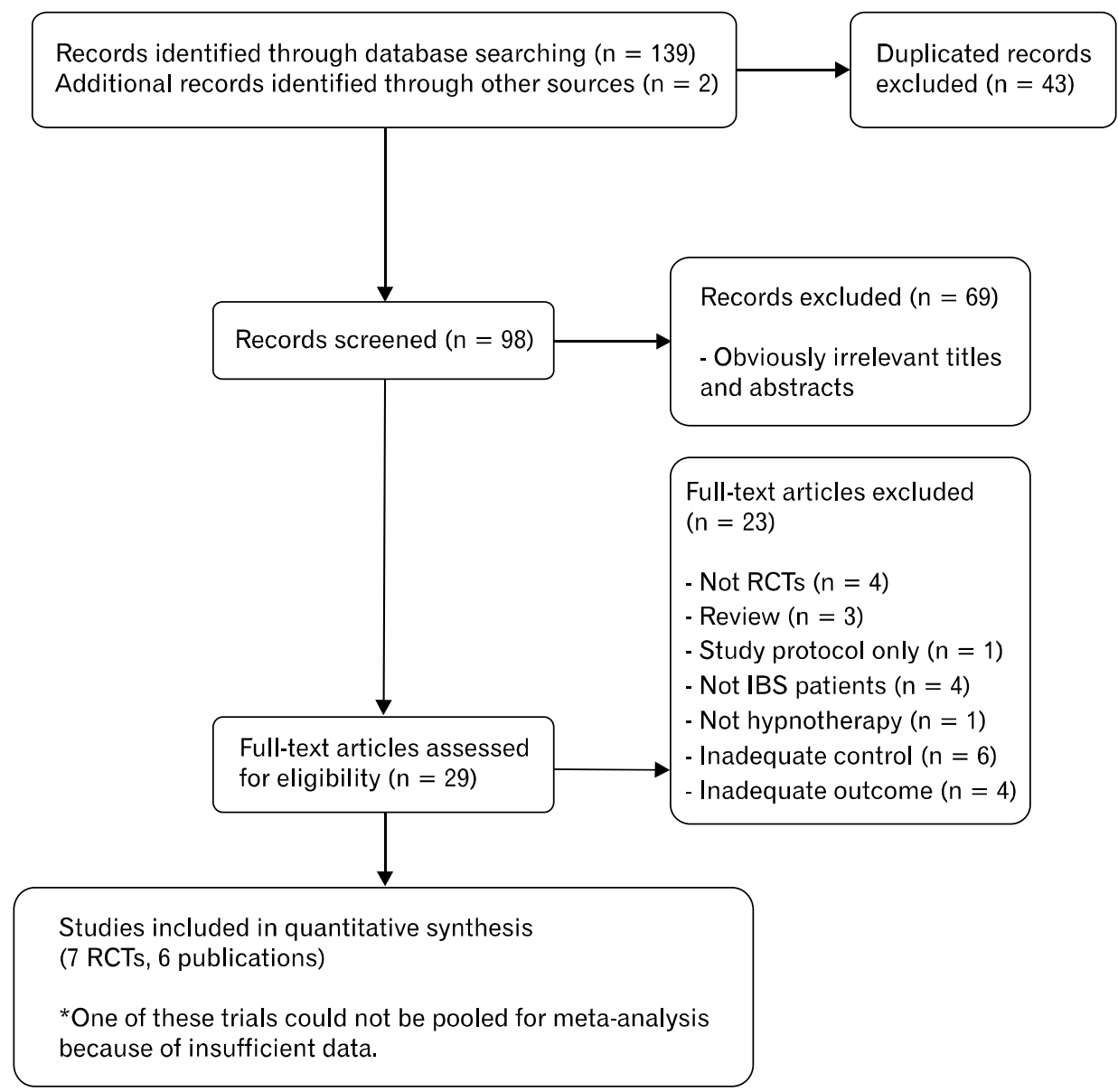

Figure 1. Flow diagram of studies identified in the systematic review. IBS, irritable bowel syndrome; RCT, randomized controlled trial. 


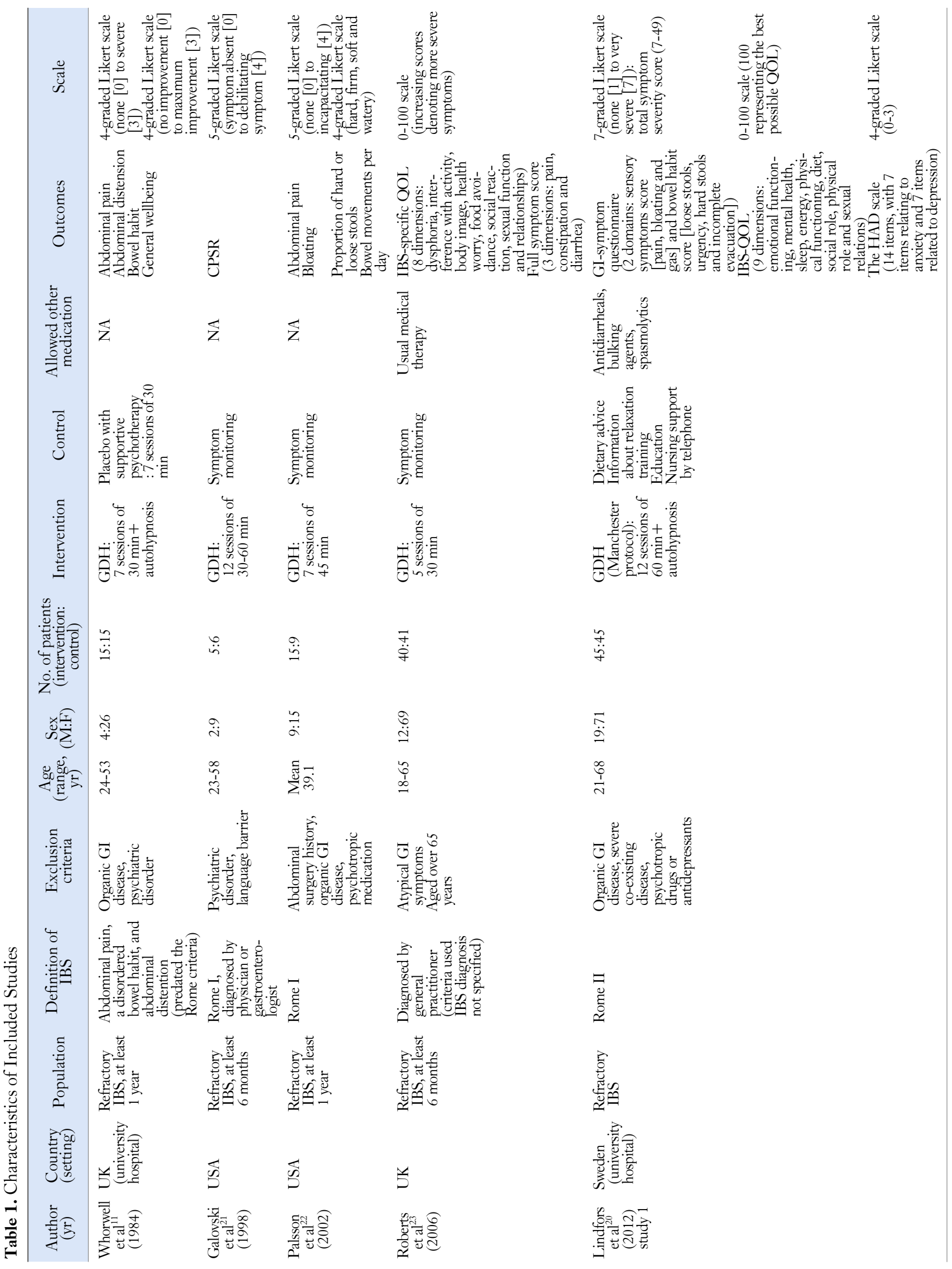


Han Hee Lee, et al

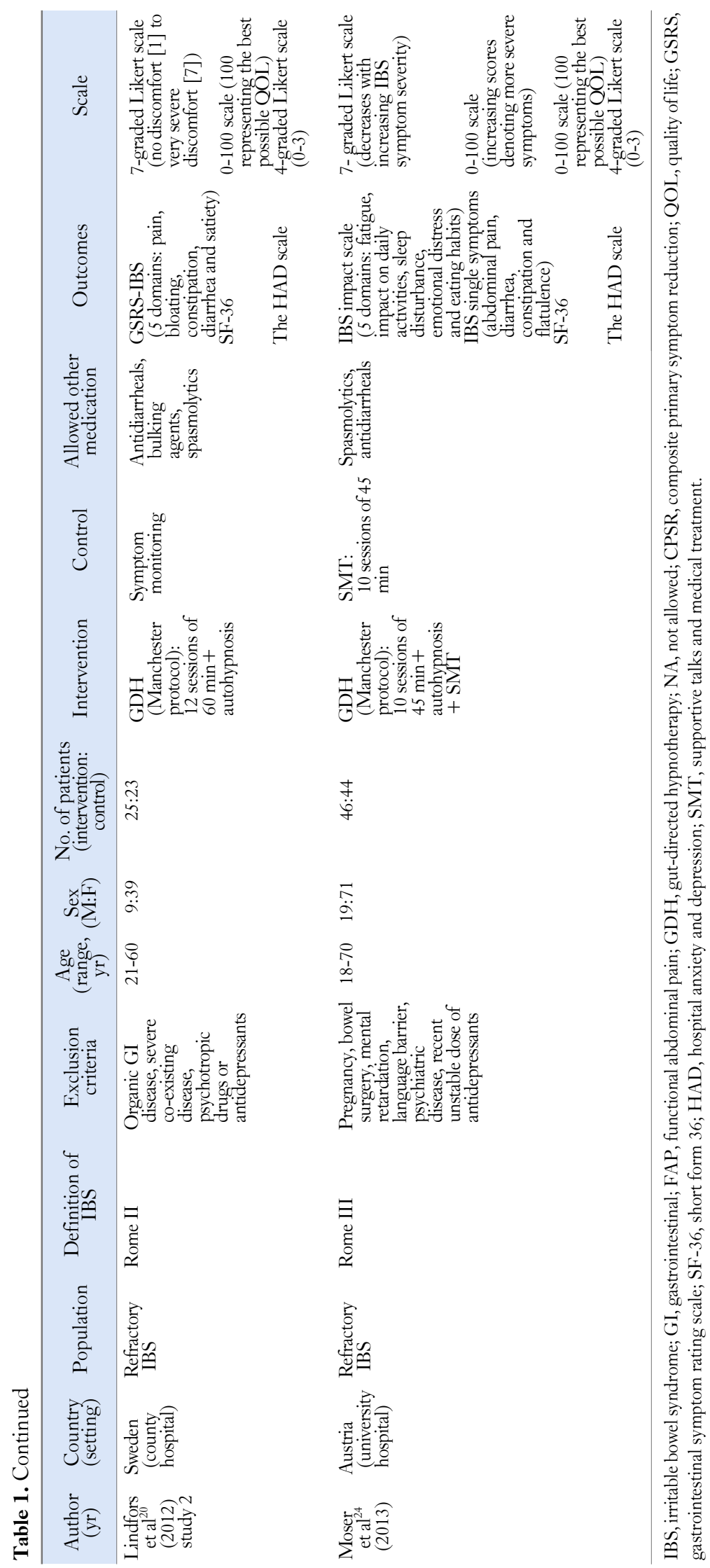


tion (SD) is needed for use of the change score in a meta-analysis. One trial presented the standard deviation of the changes in their report. $^{20}$ From these data, we calculated the correlation coefficient by using the following formula.

Corr $($ correlation coefficient $)=\frac{\mathrm{SD}_{\text {baseline }}^{2}+\mathrm{SD}_{\text {final }}^{2}-\mathrm{SD}_{\text {change }}^{2}}{2 \times \mathrm{SD}_{\text {baseline }} \times \mathrm{SD}_{\text {final }}}$

We also used the following formula to calculate the SD of the change by using the correlation coefficient in the studies that did not report this value.

$$
\mathrm{SD}_{\text {change }}=\sqrt{\mathrm{SD}_{\text {baseline }}^{2}+\mathrm{SD}_{\text {final }}^{2}-\left(2 \times \text { Cor } \mathrm{r} \times \mathrm{SD}_{\text {baseline }} \times \mathrm{SD}_{\text {final }}\right)}
$$

To combine the different scales, we used the standardized mean difference (SMD) rather than the actual means because the SMD does not depend on the measurement scale. To evaluate the magnitude of the effect size calculated by SMD, Cohen's categories were used with $0.0-0.2=$ not a substantial effect size, $>$ $0.2-0.5=$ a small effect size, $>0.5-0.8=$ a medium effect size, and $>0.8=$ a large effect size. ${ }^{26}$
Meta-analysis was performed using the software Review Manager version 5.2.3 (RevMan for Windows 7; the Nordic Cochrane Center, Copenhagen, Denmark) provided by the Cochrane Collaboration. Meta-analysis was performed using a fixed-effects model and a random-effects model for each outcome, and the analyses were compared. A $P$-value $<0.05$ was considered significant.

Statistical heterogeneity among studies was assessed using the $\chi^{2}$ test, defining a significant heterogeneity as a $P$-value $<$ 0.1 , and was quantified by measuring $I^{2}$. An $I^{2}$ value $>50 \%$ suggested significant statistical heterogeneity. The fixed-effects model of meta-analysis was used in the case of statistical homogeneity, whereas in the case of statistical heterogeneity, the random-effects model was applied. Analysis and reporting followed the Preferred Reporting Items for Systematic reviews and Meta-analyses (PRISMA) guidelines. ${ }^{27}$ Forest plots of SMDs for the assessed outcomes with 95\% confidence intervals (CIs) and funnel plots were generated. The latter were assessed for evidence of asymmetry and therefore possible reporting bias or publication bias.
A

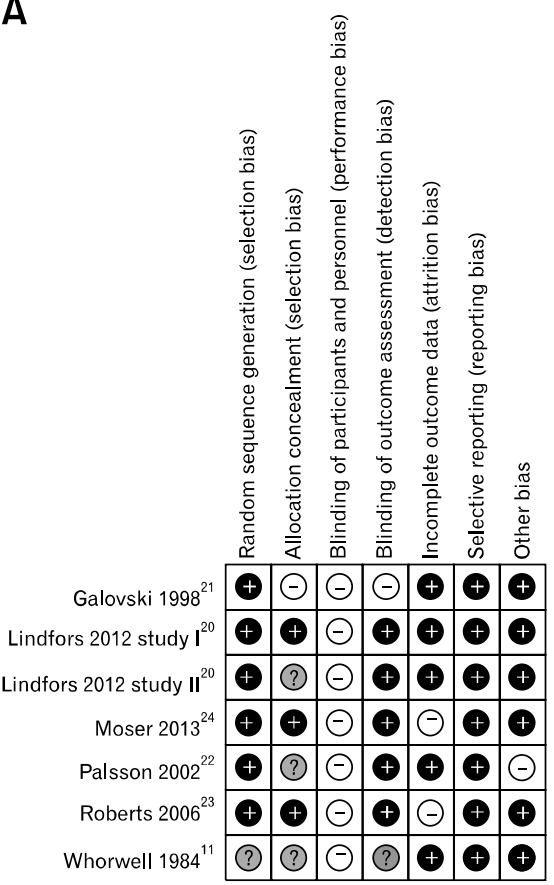

B

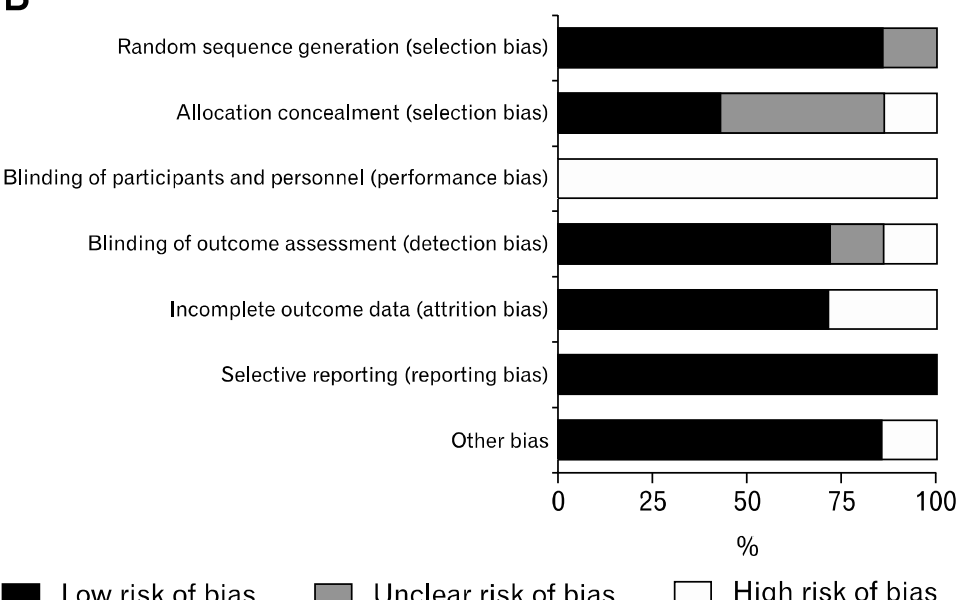

Figure 2. Assessment of risk of bias in this meta-analysis. Risk of bias summary. (A) Summary of risk of bias for each trial assessed by Cochrane Collaboration' tool, plus sign was for a judgment of Yes or low risk of bias, minus sign was for a judgment of No or high risk of bias, and question mark was for a judgment of Unclear, or uncertain risk of bias, which meant there was insufficient information to permit a judgment of Yes or No. Risk of bias graph. (B) Risk of bias graph about each risk of bias item presented as percentages across all included studies. 


\section{Results}

\section{Description of Included Studies}

The characteristics of the included studies are shown in Table 1. A total of 374 patients ( 74 men and 300 women) were included in this meta-analysis: 191 patients were in the hypnotherapy group and 183 patients were in the control group. Gut-directed hypnotherapy $(\mathrm{GDH})$ was applied to the patients as an intervention in all 7 trials. The frequency of this intervention varied from 5 to 12 sessions, and the duration of each session varied from 30 to 60 minutes. Three of the included trials used the Manchester protocol for GDH. ${ }^{20,24,28}$ Moser et al delivered GDH in group sessions. ${ }^{24}$ Four trials performed GDH with usual medical therapy or supportive talks and medical treatment. $^{20,23,24}$ Various methods were used for the control groups. Four trials monitored symptoms only in the control group, ${ }^{20-23}$ and others provided supportive and medical therapy. All trials included as outcomes IBS-related gastrointestinal symptoms. QOL was assessed in 4 trials. ${ }^{20,23,24}$ No adverse events were reported in any trial.

\section{Risk of Bias}

The quality of RCTs included in our review was assessed by the Cochrane risk of bias tool (Fig. 2). Selection bias was shown in one trial because its method was not optimal for ensuring adequate allocation concealment. ${ }^{21}$ Performance bias was high in all trials because it was not possible to blind participants and therapists in this type of intervention. Attrition bias was high in 2 trials. ${ }^{23,24}$ Other type of bias was high in one trial. Four trials used intention-to-treat analysis, ${ }^{11,20,24}$ and the others did not.

\section{Effects}

The data for 6 of the 7 included RCTs were pooled for analysis. $^{20-24}$ The data of one study were not available for analysis after we tried unsuccessfully to correspond with the author. ${ }^{11}$

The outcomes were evaluated at 3 months for short-term effects. In the cases of short-term effects, although some studies reported their results at 2 or 4 months, we regarded them as the same period as 3 months. The long-term outcomes were measured at 1 year.

\section{Abdominal pain}

Four RCTs reported an abdominal pain change score. ${ }^{20,22-24}$
A

\begin{tabular}{lrrr} 
& \multicolumn{3}{c}{ GDH } \\
\cline { 2 - 4 } Study of subgroup & Mean & SD & Tota \\
\hline Lindfors 2012 study $2^{20}$ & -0.8 & 1.24 & 25 \\
Palsson 2012 22 & -11 & 6.21 & 15 \\
Roberts 2006 & -21.2 & 18.6 & 30
\end{tabular}

Total $(95 \% \mathrm{Cl})$

Heterogeneity: $\mathrm{Tau}^{2}=0.40 ; \mathrm{Chi}^{2}=8.87, \mathrm{df}=2(P=0.01) ; I^{2}=77 \%$

Test for overall effect: $Z=1.98(P=0.05)$

*Palsson 2002: measured at 4 months

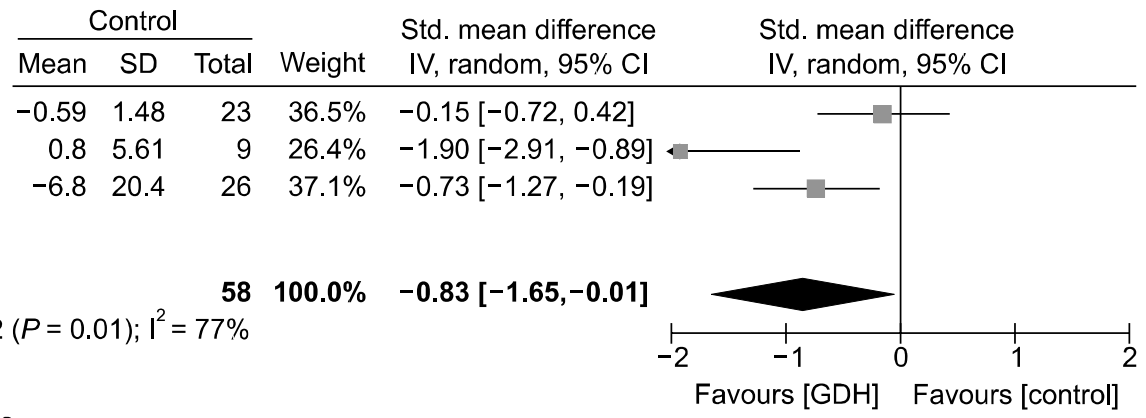

B

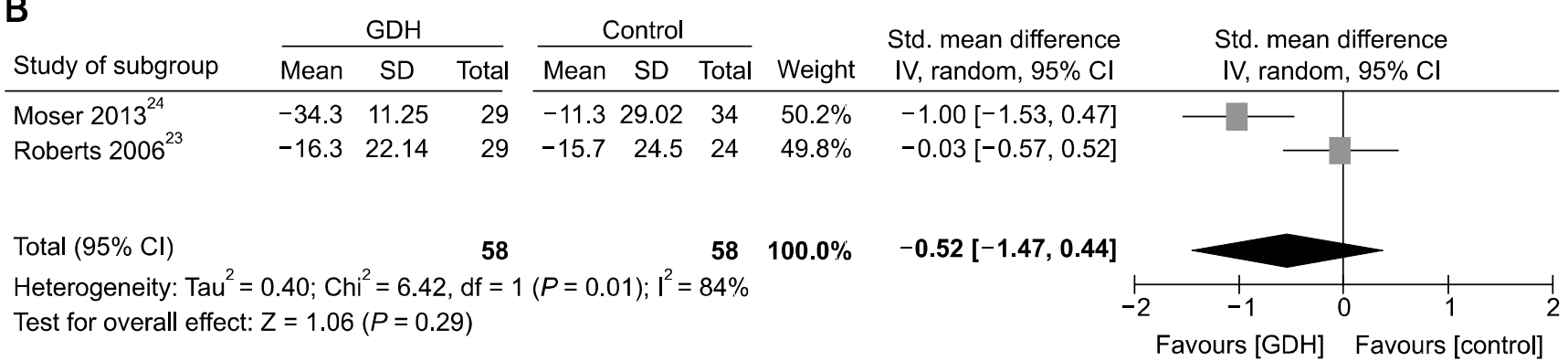

Figure 3. Forest plots of abdominal pain change score. (A) Meta-analysis of 3 months results. (B) Meta-analysis of 1-year results. GDH, gut-directed hypnotherapy; Std., standardized. 
Three reported it at 3 months, ${ }^{20,22,23}$ which showed a significant difference in favor of the GDH group (SMD -0.83; 95\% CI, -1.65 to $-0.01 ; P=0.050$ ) (Fig. $3 \mathrm{~A}$ ). The random-effects model was used because there was considerable heterogeneity $\left(I^{2}=\right.$ $77 \%, P=0.010)$. Based on Cohen's categories, the effect size of the abdominal pain change score at 3 months was large. The ab- dominal pain change score at 1 year was reported by 2 of the 4 RCTs. ${ }^{23,24}$ Meta-analysis using the fixed-effects model showed a significant difference in favor of the GDH group (SMD, -0.53; $95 \% \mathrm{CI},-0.90$ to $-0.15 ; P=0.006)$. However, there was considerable heterogeneity $\left(I^{2}=84 \%, P=0.010\right)$. When the randomeffects model was used, there was no significant difference

A

\begin{tabular}{|c|c|c|c|c|}
\hline \multirow{2}{*}{ Study of subgroup } & \multicolumn{3}{|c|}{ GDH } & \\
\hline & Mean & SD & $\overline{\text { Total }}$ & Mean \\
\hline Lindfors 2012 study $2^{20}$ & -0.3 & 1.59 & 25 & -0.11 \\
\hline Roberts $2006^{23}$ & -9.5 & 21.57 & 34 & -7.1 \\
\hline \multirow{2}{*}{\multicolumn{5}{|c|}{$\begin{array}{l}\text { Total }(95 \% \mathrm{Cl}) \\
\text { Heterogeneity: } \mathrm{Chi}^{2}=0.00, \mathrm{df}=1(P=0.98) ;\left.\right|^{2}=0 \%\end{array}$}} \\
\hline & & & & \\
\hline \multicolumn{5}{|c|}{ Test for overall effect: $Z=0.64(P=0.52)$} \\
\hline
\end{tabular}

Control

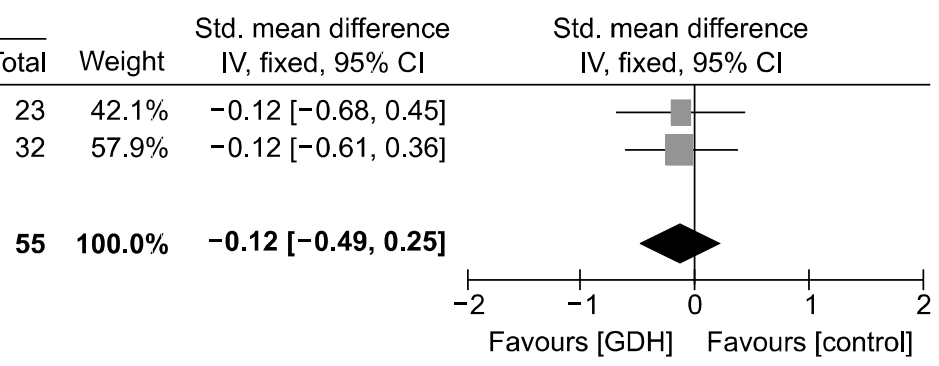

B

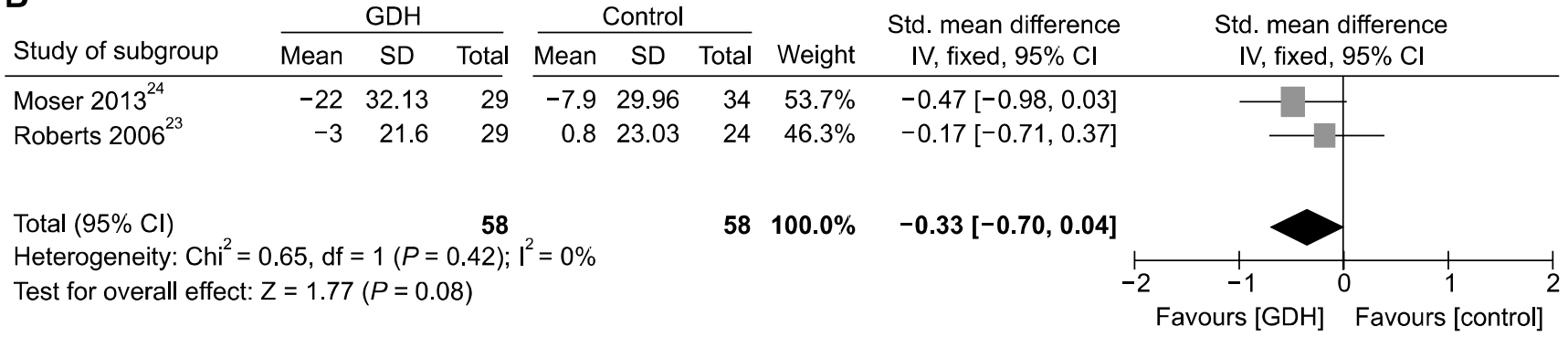

C

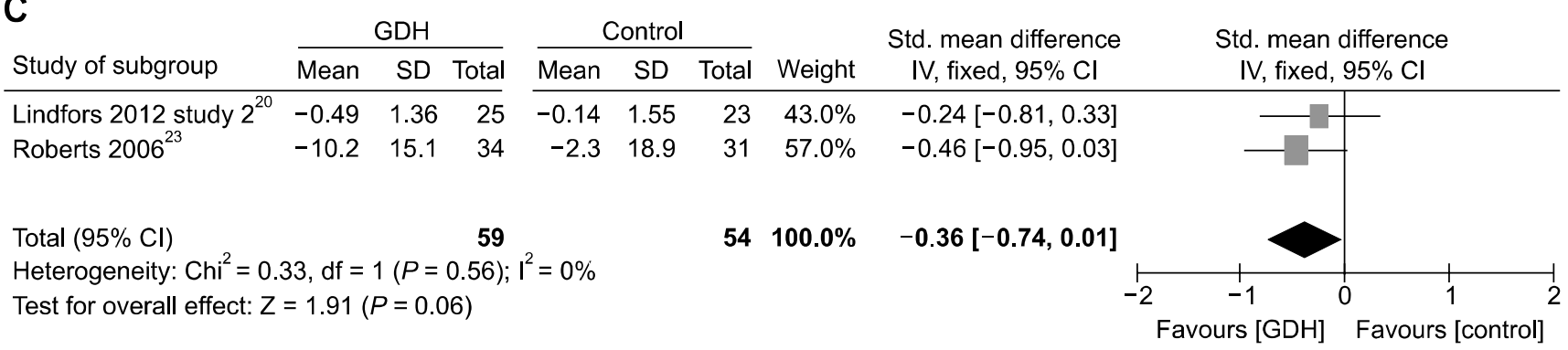

D

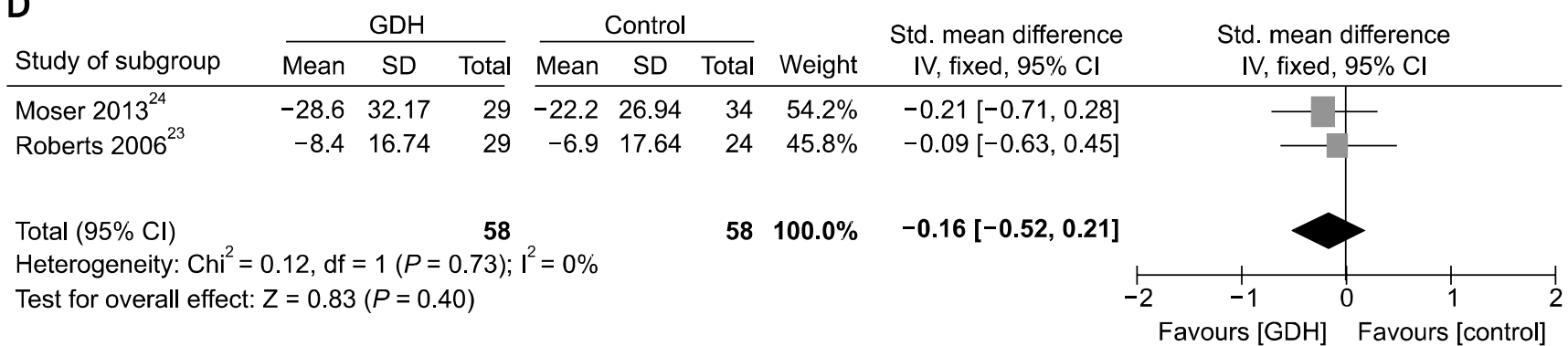

Figure 4. Forest plots of constipation change score at 3 months (A) and 1 year (B), diarrhea change score at 3 months (C) and 1 year (D). GDH, gut-directed hypnotherapy; Std., standardized. 
Table 2. Change in Overall Gastrointestinal Symptom Score

\begin{tabular}{|c|c|c|c|c|c|c|c|}
\hline \multirow{2}{*}{ Author (yr) } & \multirow{2}{*}{ Outcome measurement } & \multicolumn{2}{|c|}{3 months } & \multirow{2}{*}{$P$-value } & \multicolumn{2}{|c|}{12 months } & \multirow{2}{*}{$P$-value } \\
\hline & & Intervention (SD) & Control (SD) & & Intervention (SD) & Control (SD) & \\
\hline Galovski et al ${ }^{21}$ (1998) & $\mathrm{CPSR}^{\mathrm{a}}$ & $-0.55(0.53)$ & $0.32(0.49)$ & 0.00047 & NA & NA & NA \\
\hline Roberts et $\mathrm{al}^{23}(2006)$ & Full symptom score & $-13.00(10.50)$ & $-4.5(13.90)$ & 0.008 & $-9.10(14.00)$ & $-6.40(14.70)$ & 0.440 \\
\hline Lindfors et $\mathrm{al}^{20}$ (2012) study & GI-symptom questionnaire & $-4.50(8.60)$ & $-0.80(7.30)$ & $<0.05$ & NA & NA & $\mathrm{NA}$ \\
\hline Lindfors et $\mathrm{al}^{20}$ (2012) study 2 & GSRS-IBS & $-0.43(0.90)$ & $-0.10(1.00)$ & 0.220 & NA & NA & NA \\
\hline
\end{tabular}

${ }^{a}$ CPSR was measured at right after end of treatment.

SD, standard deviation; CPSR, composite primary symptom reduction; NA, not allowed; GSRS, gastrointestinal symptom rating scale; IBS, irritable bowel syndrome.

(SMD, $-0.52 ; 95 \% \mathrm{CI},-1.47$ to $0.44 ; P=0.290$ ) (Fig. 3B).

\section{Constipation and diarrhea}

The constipation change score was reported by $3 \mathrm{RCTs} .^{20,23,24}$ Two of these 3 trials reported this score at 3 months, ${ }^{20,23}$ and this value did not change significantly (SMD, $-0.12 ; 95 \% \mathrm{CI},-0.49$ to $0.25 ; P=0.520$ ) (Fig. $4 \mathrm{~A}$ ). The constipation change score at 1 year was reported by 2 of the 3 RCTs. ${ }^{23,24}$ Meta-analysis showed no significant difference (SMD, $-0.33 ; 95 \% \mathrm{CI},-0.70$ to 0.04; $P=0.080$ ) (Fig. 4B). Meta-analysis of the diarrhea change score at 3 months was reported by 2 RCTs, ${ }^{20,23}$ and there was no significant difference (SMD, $-0.36 ; 95 \% \mathrm{CI},-0.74$ to $0.01 ; P=$ 0.060 ) (Fig. 4C). The diarrhea change score at 1 year was reported by 2 RCTs, ${ }^{23,24}$ and the meta-analysis showed no significant difference (SMD, -0.16 ; $95 \% \mathrm{CI},-0.52$ to $0.21 ; P=$ 0.400) (Fig. 4D).

\section{Overall gastrointestinal symptoms}

Four trials attempted to measure the overall gastrointestinal symptom score in IBS patients (Table 2). Different studies used different assessment tools, and these studies were not eligible to be combined for the meta-analysis. Three trials showed that the hypnotherapy group had greater improvement in the overall gastrointestinal symptom score at 3 months compared with the control group, whereas the difference in the symptom score did not differ significantly between the 2 groups in the other trial. Only one trial reported the result at 1 year, and there was no significant difference between the hypnotherapy group and control group. ${ }^{23}$ The studies by Lindfors et $\mathrm{al}^{20}$ (studies 1 and 2 in Table 2) did not report the overall gastrointestinal symptom scores at 12 months in the control group because it was considered unethical to not allow the controls to receive hypnotherapy available in their clinical settings. Thus, we could not compare the change in overall gastrointestinal symptom scores at 12 months between the hypnotherapy and control groups.

\section{Quality of life}

Four RCTs measured QOL. ${ }^{20,23,24}$ Two used a generic health-related QOL measure (short-form 36 health survey [SF-36]). ${ }^{20,24}$ Study 2 of Lindfors et $\mathrm{al}^{20}$ showed no significant differences in SF-36 scores between the GDH group and control group. Moser et $\mathrm{al}^{24}$ reported a significant improvement in QOL scores at 3 months and 1 year in 4 dimensions (role physical, general health, vitality and social functioning) and at 1 year in 3 more dimensions (physical functioning, bodily pain and mental health) compared with the control groups.

Three trials used disease-specific QOL instruments. ${ }^{20,23,24}$ Roberts et al used an IBS-specific QOL measure ${ }^{13}$ and found no significant difference in QOL scores between the hypnotherapy and control groups at 3 months and 1 year. ${ }^{23}$ Study 1 of Lindfors et $\mathrm{al}^{20}$ used an IBS-specific QOL measure ${ }^{29}$ and reported a significant improvement at 3 months in the GDH group in the dimensions of mental health, sleep, energy and social role versus baseline. $^{20}$ This improvement was maintained significantly at 1 year, and additional improvement in emotional functioning was identified. However, there was no significant difference in the changes in QOL at 3 months between the GDH group and control group. Moser et $\mathrm{al}^{24}$ used an IBS-impact scale, a disease-specific documentation of the impact of IBS on patients' lives. ${ }^{30}$ In this study, the hypnotherapy group showed a significant improvement in IBS-impact scale scores at 3 months and 1 year compared with the control group.

\section{Discussion}

We reviewed 7 RCTs with a total of 374 patients that compared hypnotherapy with various control treatments to examine whether hypnotherapy as treatment for IBS would have sig- 
nificant beneficial effects on gastrointestinal symptoms and QOL. This review is the first study to conduct a meta-analysis of the efficacy of hypnotherapy in IBS patients. This meta-analysis revealed that hypnotherapy significantly improved abdominal pain, at least at short-term follow-up. Hypnotherapy also provided benefit for overall gastrointestinal symptoms. However, evidence for the long-term efficacy of hypnotherapy is lacking because of an insufficient number of studies.

Our search strategy was comprehensive in that we used 4 databases, distinct inclusion criteria and the Cochrane risk of bias tool for each trial. The previous reviews included insufficient evidences because of the small number of RCTs and methodological flaws. ${ }^{17,18}$ Our study provides more reliable evidence by including 3 recent well-designed RCTs.

Randomization is especially important in trials of fluctuating diseases such as IBS, since recruitment of patients to a trial usually occurs during a period of increased symptomatology. ${ }^{17}$ The current study included only RCTs whose aim was to establish the effectiveness of hypnotherapy. The calculation of a summary measure of effect had been difficult because of heterogeneity in the outcome measures in the previous reviews. Likert scales of different grades were used to obtain scores for abdominal pain and symptoms of defecatory dysfunction in individual trials. In this meta-analysis, we used the SMD as a summary statistic for studies that assessed the same outcome but measured it with different methods.

In this meta-analysis, the potential benefits of hypnotherapy in treating constipation and diarrhea were not verified because of insufficient power and internal validity. In addition, patient-reported ratings of changes in bowel habits were not able to delineate adequately whether any benefits were achieved in all of the important subconcepts (i.e., urgency, stool consistency and stool frequency). Further trials should include a predefined definition and grade for bowel habits such as the British stool scale to evaluate benefits precisely. ${ }^{31}$

GDH involves hypnotic induction by using a variety of techniques, including progressive relaxation, followed by creating imagery related to symptom control and normalization of gut function. The mechanism through which hypnotherapy improves abdominal pain in IBS patients is not well understood. Hypnotic reduction of somatic pain is thought to reduce the activation of certain areas of the brain, which appears to be exaggerated in IBS. $^{32}$ It is also assumed that hypnotherapy normalizes visceral sensation, decreases colonic phasic contractions, and reverses negative thoughts of IBS patients about their condition. ${ }^{33-35}$
Although 3 more RCTs were added in this meta-analysis after the last systematic review, the number of included studies was too small to draw firm conclusions. The control groups varied somewhat between studies, from no treatment to supportive therapy; however, separate comparisons of the different control treatments were not conducted because of the limited number of included trials. Difficulties in blinding because of the nature of the intervention also contribute to the potential for performance bias.

In conclusion, hypnotherapy may be a useful and safe therapeutic option for refractory IBS in short term. More high-quality RCTs are needed for evaluating the long-term efficacy of hypnotherapy. All of the included studies targeted refractory IBS; therefore, the beneficial effects of hypnotherapy cannot be generalized to all IBS patients.

\section{References}

1. Thompson WG, Longstreth GF, Drossman DA, Heaton KW, Irvine EJ, Muller-Lissner SA. Functional bowel disorders and functional abdominal pain. Gut 1999;45(suppl 2):II43-II47.

2. Karabulut GS, Beşer OF, Erginöz E, Kutlu T, Cokuğraş FÇ, Erkan $\mathrm{T}$. The Incidence of Irritable Bowel Syndrome in Children Using the Rome III Criteria and the Effect of Trimebutine Treatment. J Neurogastroenterol Motil 2013;19:90-93.

3. Agrawal A, Whorwell PJ. Irritable bowel syndrome: diagnosis and management. BMJ 2006;332:280-283.

4. Whitehead WE, Burnett CK, Cook EW 3rd, Taub E. Impact of irritable bowel syndrome on quality of life. Dig Dis Sci 1996;41: 2248-2253.

5. Gralnek IM, Hays RD, Kilbourne A, Naliboff B, Mayer EA. The impact of irritable bowel syndrome on health-related quality of life. Gastroenterology 2000;119:654-660.

6. Quartero AO, Meineche-Schmidt V, Muris J, Rubin G, de Wit N. Bulking agents, antispasmodic and antidepressant medication for the treatment of irritable bowel syndrome. Cochrane Database Syst Rev 2005:(2):CD003460.

7. Francis CY, Whorwell PJ. Bran and irritable bowel syndrome: time for reappraisal. Lancet 1994;344:39-40.

8. Poynard T, Naveau S, Mory B, Chaput JC. Meta-analysis of smooth muscle relaxants in the treatment of irritable bowel syndrome. Aliment Pharmacol Ther 1994;8:499-510.

9. Drossman DA, Camilleri M, Mayer EA, Whitehead WE. AGA technical review on irritable bowel syndrome. Gastroenterology 2002; 123:2108-2131.

10. Lackner JM, Mesmer C, Morley S, Dowzer C, Hamilton S. Psychological treatments for irritable bowel syndrome: a systematic review and meta-analysis. J Consult Clin Psychol 2004;72:1100-1113.

11. Whorwell PJ, Prior A, Faragher EB. Controlled trial of hypnotherapy in the treatment of severe refractory irritable-bowel syndrome. Lancet 1984;2:1232-1234.

12. Whorwell PJ, Prior A, Colgan SM. Hypnotherapy in severe irritable bowel syndrome: further experience. Gut 1987;28:423-425. 
13. Harvey RF, Hinton RA, Gunary RM, Barry RE. Individual and group hypnotherapy in treatment of refractory irritable bowel syndrome. Lancet 1989;1:424-425.

14. Houghton LA, Heyman DJ, Whorwell PJ. Symptomatology, quality of life and economic features of irritable bowel syndrome - the effect of hypnotherapy. Aliment Pharmacol Ther 1996;10:91-95.

15. Gonsalkorale WM, Houghton LA, Whorwell PJ. Hypnotherapy in irritable bowel syndrome: a large-scale audit of a clinical service with examination of factors influencing responsiveness. Am J Gastroenterol 2002;97:954-961.

16. Gonsalkorale WM, Miller V, Afzal A, Whorwell PJ. Long term benefits of hypnotherapy for irritable bowel syndrome. Gut 2003;52: 1623-1629.

17. Wilson S, Maddison T, Roberts L, Greenfield S, Singh S; Birmingham IBS Research Group. Systematic review: the effectiveness of hypnotherapy in the management of irritable bowel syndrome. Aliment Pharmacol Ther 2006;24:769-780.

18. Webb AN, Kukuruzovic RH, Catto-Smith AG, Sawyer SM. Hypnotherapy for treatment of irritable bowel syndrome. Cochrane Database Syst Rev 2007:(4):CD005110.

19. National Collaborating Center for Nursing and Supportive Care (UK). Irritable bowel syndrome in adults: diagnosis and management of irritable bowel syndrome in primary care. London: Royal College of Nursing (UK) 2008.

20. Lindfors $P$, Unge $P$, Arvidsson $P$, et al. Effects of gut-directed hypnotherapy on IBS in different clinical settings-results from two randomized, controlled trials. Am J Gastroenterol 2012;107:276-285.

21. Galovski TE, Blanchard EB. The treatment of irritable bowel syndrome with hypnotherapy. Appl Psychophysiol Biofeedback 1998; 23:219-232.

22. Palsson OS, Turner MJ, Johnson DA, Burnett CK, Whitehead WE. Hypnosis treatment for severe irritable bowel syndrome: investigation of mechanism and effects on symptoms. Dig Dis Sci 2002;47: 2605-2614.

23. Roberts L, Wilson S, Singh S, Roalfe A, Greenfield S. Gut-directed hypnotherapy for irritable bowel syndrome: piloting a primary carebased randomised controlled trial. Br J Gen Pract 2006;56:115-121.
24. Moser G, Trägner S, Gajowniczek EE, et al. Long-term success of GUT-directed group hypnosis for patients with refractory irritable bowel syndrome: a randomized controlled trial. Am J Gastroenterol 2013;108:602-609.

25. Higgins JPT, Green S. Cochrane handbook for systematic reviews of interventions: version 5.1.0. 2011. Available from URL: http://www. cochrane-handbook.org (accessed 26 January, 2014)

26. Cohen J. Statistical power analysis for the behavioral sciences. 2nd ed. Hillsdale (NJ): Lawrence Erlbaum Associates 1988

27. Moher D, Liberati A, Tetzlaff J, Altman DG. Preferred reporting items for systematic reviews and meta-analyses: the PRISMA statement. Ann Intern Med 2009;151:264-269.

28. Vlieger AM, Menko-Frankenhuis C, Wolfkamp SC, Tromp E, Benninga MA. Hypnotherapy for children with functional abdominal pain or irritable bowel syndrome: a randomized controlled trial. Gastroenterology 2007;133:1430-1436.

29. Hahn BA, Kirchdoerfer LJ, Fullerton S, Mayer E. Evaluation of a new quality of life questionnaire for patients with irritable bowel syndrome. Aliment Pharmacol Ther 1997;11:547-552.

30. Longstreth GF, Bolus R, Naliboff B, et al. Impact of irritable bowel syndrome on patients' lives: development and psychometric documentation of a disease-specific measure for use in clinical trials. Eur J Gastroenterol Hepatol 2005;17:411-420.

31. Lewis SJ, Heaton KW. Stool form scale as a useful guide to intestinal transit time. Scand J Gastroenterol 1997;32:920-924.

32. Rainville P, Duncan GH, Price DD, Carrier B, Bushnell MC. Pain affect encoded in human anterior cingulate but not somatosensory cortex. Science 1997;277:968-971.

33. Whorwell PJ, Houghton LA, Taylor EE, Maxton DG. Physiological effects of emotion: assessment via hypnosis. Lancet 1992;340:69-72.

34. Lea R, Houghton LA, Calvert EL, et al. Gut-focused hypnotherapy normalizes disordered rectal sensitivity in patients with irritable bowel syndrome. Aliment Pharmacol Ther 2003;17:635-642.

35. Gonsalkorale WM, Toner BB, Whorwell PJ. Cognitive change in patients undergoing hypnotherapy for irritable bowel syndrome. J Psychosom Res 2004;56:271-278. 